\title{
Circular RNA-ITCH Suppresses Lung Cancer Proliferation via Inhibiting the Wnt/ $\beta$-Catenin Pathway
}

\author{
Li Wan, Lin Zhang, Kai Fan, Zai-Xing Cheng, Quan-Chao Sun, and Jian-Jun Wang \\ Department of Thoracis Surgery, Union Hospital, Tongji Medical College, Huazhong University of Science and Technology, \\ Wuhan 430022, China \\ Correspondence should be addressed to Jian-Jun Wang; wangjianjun1961@163.com
}

Received 1 April 2016; Revised 28 June 2016; Accepted 25 July 2016

Academic Editor: Renato Franco

Copyright (C) 2016 Li Wan et al. This is an open access article distributed under the Creative Commons Attribution License, which permits unrestricted use, distribution, and reproduction in any medium, provided the original work is properly cited.

As a special form of noncoding RNAs, circular RNAs (circRNAs) played important roles in regulating cancer progression mainly by functioning as miRNA sponge. While the function of circular RNA-ITCH (cir-ITCH) in lung cancer is still less reported, in this study, we firstly detected the expression of cir-ITCH in tumor tissues and paired adjacent noncancer tissues of 78 patients with lung cancer using a TaqMan-based quantitative real-time PCR (qRT-PCR). The results showed that the expression of $c$ ir-ITCH was significantly decreased in lung cancer tissues. In cellular studies, cir-ITCH was also enhanced in different lung cancer cell lines, A549 and NIC-H460. Ectopic expression of cir-ITCH markedly elevated its parental cancer-suppressive gene, ITCH, expression and inhibited proliferation of lung cancer cells. Molecular analysis further revealed that cir-ITCH acted as sponge of oncogenic miR-7 and miR-214 to enhance ITCH expression and thus suppressed the activation of Wnt/ $\beta$-catenin signaling. Altogether, our results suggested that cir-ITCH may play an inhibitory role in lung cancer progression by enhancing its parental gene, ITCH, expression.

\section{Introduction}

Lung cancer is the most common incident cancer and the leading cause of cancer-related death in China [1]. Although continuous efforts have been devoted to improving the therapeutic response and treatments for stage I lung cancer have demonstrated survival benefits $[2,3]$, the overall five-year survival rate of advanced lung cancer is still less than 15\% [46]. Therefore, the development of finding novel therapeutic targets is of particular importance for the treatments of lung cancers, and a further understanding of the molecular mechanisms underlying lung cancer is essential to achieve this goal.

Circular RNAs (circRNAs) represent a large class of endogenous RNAs with covalently closed continuous loop [7]. For decades, circRNAs were mostly misinterpreted as splicing errors that result from splicing artefacts or gene rearrangements [8]. But recently (from 2012/2013), circRNAs were rediscovered from RNA sequencing (RNA-seq) data and shown to be ubiquitous in mammalian cells and more abundant (certain circRNAs are up to 200 times) than their linear counterparts $[9,10]$. Tissue, as well as development-specific expression of circRNAs, further indicates that they originate from nonrandom back-splice events $[7,11]$. With regard to their function, several studies reported that circRNAs mainly serve as miRNA sponges to regulate gene expression $[7,12]$. For at least one specific circRNA, ciRS-7, which harbors more than 70 conventional miR-7 binding sites, impairs the regulatory effect of miR-7 in vivo [12]. miRNAs regulate a variety of essential biological functions such as cellular differentiation, apoptosis, and proliferation and thus play critical role in cancer progression [13]. Based on these clues, circRNAs were found to be closely related to development of different cancers, including esophageal squamous cell carcinoma, colorectal cancer, gastric cancer, and ovarian cancer [14-17]. Specifically, Hsa_circ_002059 expression levels are significantly correlated with distal metastasis and TNM stage of gastric cancer and thus may be a potential novel and stable biomarker for the clinical diagnosis of gastric cancer [17].

Aberrant activation of the Wnt/ $\beta$-catenin pathway plays a critical role in tumor initiation, progression, and metastasis of lung cancer [18-20]. The E3 ubiquitin (Ub) protein ligase (ITCH) inhibits Wnt $/ \beta$-catenin signaling in cancers mainly by promoting the ubiquitination and degradation of phosphorylated disheveled 2 (Dvl2) [21]. Circular RNA-ITCH 
TABLE 1: Baseline demographic and clinical characteristics of study populations.

\begin{tabular}{|c|c|c|c|c|}
\hline \multirow{2}{*}{ Characteristics } & \multicolumn{2}{|c|}{ Population } & \multirow{2}{*}{ cir-ITCH relative expression ${ }^{\#}$} & \multirow{2}{*}{$p$ value } \\
\hline & $N$ & $(\%)$ & & \\
\hline \multicolumn{5}{|l|}{ Age (years) } \\
\hline$\leq 40$ & 10 & 12.82 & $0.0607 \pm 0.0169$ & \multirow{3}{*}{$0.0076^{*}$} \\
\hline $40-60$ & 31 & 39.74 & $0.0466 \pm 0.0307$ & \\
\hline$\geq 60$ & 37 & 47.44 & $0.0357 \pm 0.0155$ & \\
\hline \multicolumn{5}{|l|}{$\operatorname{Sex}$} \\
\hline Male & 45 & 57.7 & $0.0432 \pm 0.0257$ & \multirow{2}{*}{0.9704} \\
\hline Female & 33 & 42.3 & $0.0434 \pm 0.0225$ & \\
\hline \multicolumn{5}{|l|}{ Family history } \\
\hline Yes & 23 & 29.49 & $0.0441 \pm 0.0290$ & \multirow{2}{*}{0.8437} \\
\hline No & 55 & 70.51 & $0.0429 \pm 0.0221$ & \\
\hline \multicolumn{5}{|l|}{ Smoking } \\
\hline Never & 53 & 67.95 & $0.0487 \pm 0.0283$ & \multirow{2}{*}{0.1713} \\
\hline Ever & 25 & 32.05 & $0.0407 \pm 0.0230$ & \\
\hline \multicolumn{5}{|l|}{ Drinking } \\
\hline Never & 33 & 42.3 & $0.0478 \pm 0.2352$ & \multirow{2}{*}{0.1568} \\
\hline Ever & 45 & 57.7 & $0.0399 \pm 0.0244$ & \\
\hline \multicolumn{5}{|l|}{ Tumor type } \\
\hline Adenocarcinoma & 29 & 37.18 & $0.0443 \pm 0.0267$ & \multirow{3}{*}{0.9183} \\
\hline Large cell carcinoma & 25 & 32.05 & $0.0436 \pm 0.0250$ & \\
\hline Squamous cell carcinoma & 24 & 30.77 & $0.0416 \pm 0.0218$ & \\
\hline \multicolumn{5}{|l|}{ Stage } \\
\hline I & 18 & 23.08 & $0.0605 \pm 0.0273$ & \multirow{4}{*}{$0.0011^{*}$} \\
\hline II & 21 & 26.92 & $0.0444 \pm 0.0260$ & \\
\hline III & 22 & 28.21 & $0.0375 \pm 0.0182$ & \\
\hline IV & 17 & 21.79 & $0.0309 \pm 0.0142$ & \\
\hline
\end{tabular}

${ }^{*} p<0.05$ means statistically significant difference existed within subgroups.

\# Relative expression value was normalized to GAPDH expression level.

(cir-ITCH) shared some miRNAs binding sites with the $3^{\prime}$ untranslated region (UTR) of ITCH, including those for miR7, miR-17, miR-214, miR-128, and miR-216b [7, 12, 15]. As sponge of oncogenic miR-7, miR-17, and miR-214, cir-ITCH increases the level of ITCH and thus indirectly inhibits the activation of $\mathrm{Wnt} / \beta$-catenin pathway; these effects finally result in the suppression of esophageal squamous cell carcinoma [16] and colorectal cancer [15]. However, there are no reported studies on the functional roles of cir-ITCH in lung cancer.

As two oncogenic miRNAs, miR-7 and miR-214 are overexpressed in lung cancer cells, enhance radiotherapy response, and promote the progression of lung cancer [22, 23]. Thus, in this study, we hypothesized that cir-ITCH might compete with ITCH to bind to miR-7 and miR-214 and may be involved in lung cancer development. To address this hypothesis, we detected the expression of cir-ITCH in primary tumor tissues and different lung cancer cell lines. Then, the functional relevance of $c i r-I T C H$ with lung cancer was further examined by biochemical assays.

\section{Materials and Methods}

2.1. Participants and Tissue Samples. The study was approved by the Ethical Review Board for Research in Tongji Hospital, affiliated to Tongji Medical College of Huazhong University of Science and Technology. 78 lung cancer biopsy specimens and paired adjacent normal tissues were obtained from Department of Pathology of Tongji Hospital. Tissues were acquired and immediately stored at liquid nitrogen until use. There were no limitations on the age, sex, histology, or stage of lung cancer. The patients' characteristics were summarized in Table 1.

2.2. Cell Culture. Human lung cancer cell lines A549 and NCI-H460 were purchased from the Cell Bank of Type Culture Collection of the Chinese Academy of Sciences, Shanghai Institute of Cell Biology. Cells were cultured in DMEM medium (Gibco, CA, USA) and supplemented with $10 \%$ fetal bovine serum (Gibco), $2 \mu \mathrm{M} \mathrm{L}$-glutamine, $100 \mathrm{U} / \mathrm{mL}$ penicillin, and $100 \mu \mathrm{g} / \mathrm{mL}$ streptomycin sulfate. Cells were incubated at $37^{\circ} \mathrm{C}$ in a humidified atmosphere containing $5 \%$ $\mathrm{CO}_{2}$.

2.3. Circular RNA Plasmid Construction. Human cir-ITCH cDNA was synthesized by GeneWiz (Suzhou, China) and cloned into pcDNA3.1 (Invitrogen, CA, USA) as previously described $[15,16]$. Recombinant plasmid pcDNA3.1-cir-ITCH was verified by direct sequencing. 
TABLE 2: The sequences of primers used in this study.

\begin{tabular}{|c|c|c|c|}
\hline Gene & Forward $\left(5^{\prime}-3^{\prime}\right)$ & Reverse $\left(5^{\prime}-3^{\prime}\right)$ & Probe \\
\hline cir-ITCH & GCAGAGGCCAACACTGGAA & TCCTTGAAGCTGACTACGCTGAG & CCGTCCGGAACTATGAACAACAATGGCA \\
\hline GAPDH & CCATGACСССТTCATTGACC & TTGATTTTGGAGGGATCTCG & CTGAGAACGGGAAGCTTGTC \\
\hline $\begin{array}{l}\text { Linear } \\
\text { ITCH }\end{array}$ & TAGACCAGAACCTCTACCTCCTG & TTAAACTGCTGCATTGCTCCTTG & \\
\hline $\begin{array}{l}\text { Circular } \\
\text { ITCH }\end{array}$ & ACAGAGACAACCGAGAAACAGTG & GCCTTGATACTTGTTACCGTCGA & \\
\hline c-Myc & TTCGGGTAGTGGAAAACCAG & CAGCAGCTCGAATTTCTTCC & \\
\hline cyclinD1 & GAGGAGCAGCTCGCCAA & CTGTCAAGGTCCGGCCAGCG & \\
\hline GAPDH & GAAGGTGAAGGTCGGAGTC & GAAGATGGTGATGGGATTTC & \\
\hline
\end{tabular}

2.4. RNA Extraction and Real-Time Quantitative Polymerase Chain Reaction. Total RNA was isolated from cells and tissues using the TRIzol reagent (Invitrogen) according to the manufacturer's instructions. RNA was reversely transcribed into cDNA using First Strand cDNA Synthesis Kit (Toyobo). The relative gene expression of $c i r-I T C H$ was quantified using a real-time RT-PCR with the TaqMan probe. GAPDH was used as an internal control [24], and all reactions were performed in triplicate. The primers used for polymerase chain reaction (PCR) amplification are listed in Table 2.

2.5. RNase $R$ Digestion. The RNase $\mathrm{R}$ digestion reaction was performed following previously published procedures. The digestion and precipitation reactions were repeated twice with a ratio of $3 \mathrm{U}$ of enzyme/1 mg of RNA [25].

2.6. Transient Transfections and Luciferase Assays. A549 and NCI-H460 cells were seeded in 24 -well plates $\left(1 \times 10^{5}\right.$ cells per well) and cultured to about $70 \%$ confluence before transfection. Then, cells were transfected with $800 \mathrm{ng}$ of the reporter plasmids described above using Lipofectamine 2000 (Invitrogen). Cells were cotransfected with the miRNAs according to the manufacturer's instructions [26]. Each group included 6 replicates, and triplicate independent experiments were performed. $24 \mathrm{~h}$ after transfection, the cells were collected using $100 \mu \mathrm{L}$ passive buffer, and Renilla luciferase activity was detected using the Dual-Luciferase Reporter Assay System (Promega); the results were normalized against the activity of the Renilla luciferase gene [16].

2.7. Actinomycin D Assay. A549 and NCI-H460 cells were seeded at $5 \times 10^{4}$ cells per well in 24 -well plate overnight and then transiently transfected with 1 or 40 pmol of miRNA mimics (Ambion) using Lipofectamine 2000 with or without $40 \mathrm{pmol}$ of miRNA inhibitor as indicated. $24 \mathrm{~h}$ later, cells were then exposed to $2 \mathrm{mg} / \mathrm{L}$ actinomycin D (Sigma) for 1, 2, and $3 \mathrm{~h}$. The cells were harvested and the stability of the cir-ITCH mRNA was analyzed using quantitative reverse transcription PCR (qRT-PCR).

2.8. Western Blotting. Protein was isolated from cell lysis using Mammalian Protein Extraction Reagent (Thermo Fisher Scientific, Rockville, MD, USA). Equivalent amount of protein was loaded on 10\% SDS-PAGE gel (Invitrogen) and then transferred onto polyvinylidene difluoride (PVDF) membranes (Millipore, Billerica, MA, USA). The PVDF membranes were blocked with 5\% nonfat milk for 1 hour at $37^{\circ} \mathrm{C}$. Membranes were incubated overnight at $4^{\circ} \mathrm{C}$ with antiWnt3a antibody (1:1000 dilution, Santa Cruz Biotechnology, Santa Cruz, CA, USA), anti- $\beta$-catenin antibody ( $1: 1000$ dilution, Santa Cruz Biotechnology), or $\beta$-actin (1:5000 dilution, Abcam, Cambridge, MA, USA) and then incubated with secondary HRP-goat anti-rabbit/mouse antibodies (1:10000 dilution, Santa Cruz Biotechnology). Signals were detected using ECL detection reagent (Millipore) following the manufacturer's instructions.

2.9. Cell Viability Assay. Cell viability assay was carried out with a Cell Counting Kit-8 (Beyotime, Shanghai, China) according to the manufacturer's instructions [27]. $1 \times 10^{4}$ cells in $100 \mu \mathrm{L}$ of A549 and NCI-H460 cells were seeded into 96-well plates (BD Biosciences), respectively. $24 \mathrm{~h}$ after transfection, cells were incubated for another 1, 2, and 3 days. The numbers of cells per well were detected by the absorbance $(450 \mathrm{~nm})$ of reduced WST- 8 at the indicated time points. The absorbance $(450 \mathrm{~nm})$ was measured by using SpectraMax ${ }^{\circledR}$ i3x microplate reader (Molecular Devices, Sunnyvale, CA, USA). There were 6 replicates for each group, and the experiments were repeated at least 3 times.

2.10. Statistical Analysis. All data are presented as mean \pm SD and analyzed by using the GraphPad Prism version 5.00 software (GraphPad Software, CA, USA). Spearman correlation test was used to assess the association between cirITCH expression and the mRNA expression of ITCH in lung cancer tissues. Comparison between two groups for statistical significance was performed with two-tailed Student's $t$-test. For more groups, one-way ANOVA followed by NewmanKeuls post hoc test was used. $p<0.05$ was considered statistically significant.

\section{Results}

3.1. Identification of the Circular RNA. We designed two sets of primers for ITCH detection: a divergent set that was expected to amplify only the circular form and an oppositedirected set to amplify the linear forms. cDNA and genomic DNA were used as templates. The circular form, namely, cirITCH, was amplified by using the divergent primers on cDNA (Figure 1(a), upper panel). And as expected, there was no 


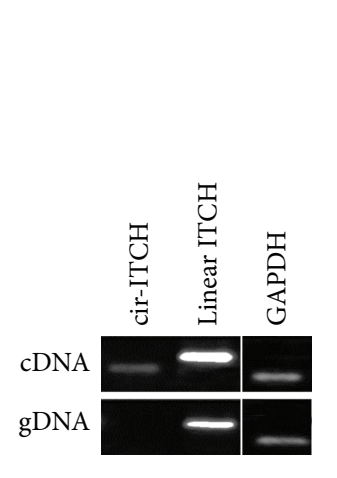

(a)

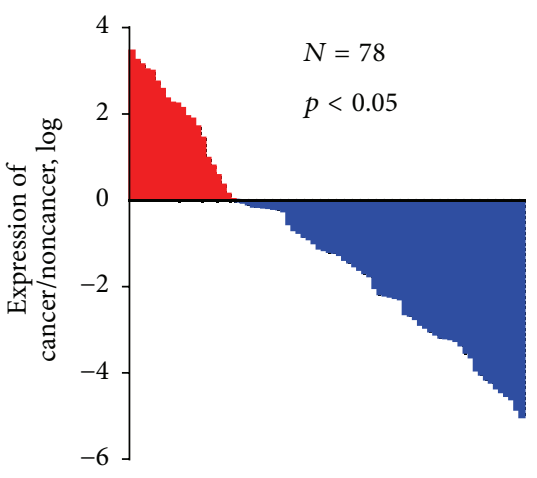

(b)

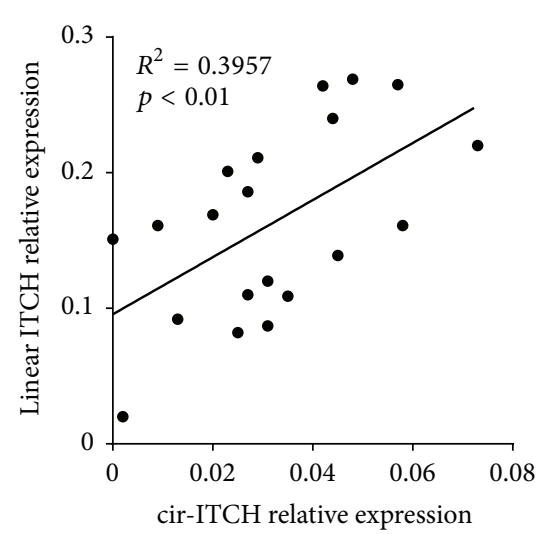

(c)

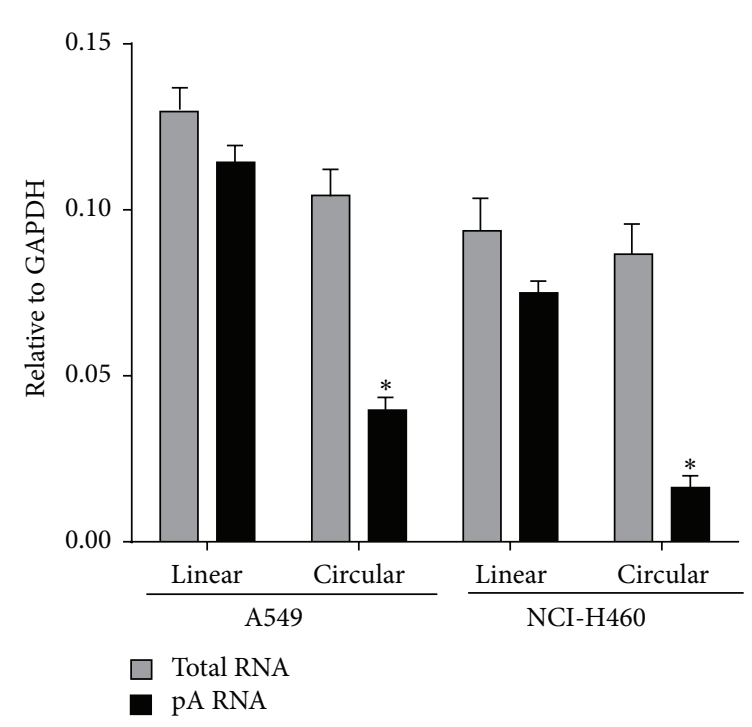

(d)

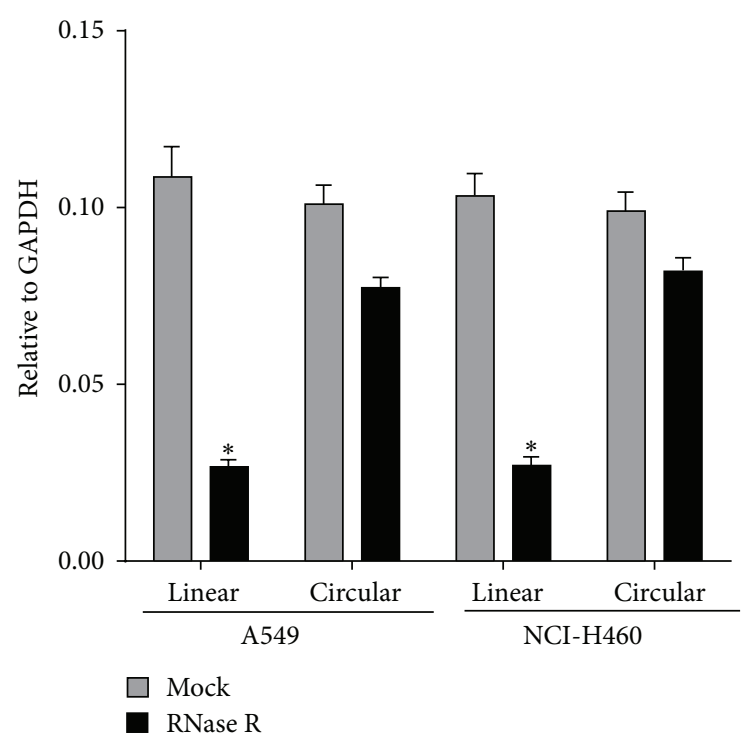

(e)

FIGURE 1: The expression level of cir-ITCH is closely related to lung cancer. (a) cir-ITCH was amplified by RT-PCR with divergent primers in cDNA but was not amplified in genomic DNA (gDNA). GAPDH, linear control. (b) qRT-PCR based on TaqMan probe was used to analyze the expression level of cir-ITCH in lung cancer tissues and paired noncancerous tissues. GAPDH was used as endogenous control. (c) The linear correlations between the cir-ITCH expression levels and linear ITCH were tested by Spearman analysis. The relative expression value was normalized by GAPDH expression level. (d) Random primers and oligo dT primers were used, respectively, in the reverse transcription experiments. The predicted circular RNA is absent in poly(A)-enriched samples. (e) The predicted circular RNA is resistant to RNase R treatment. Data are presented as mean \pm SEM from three independent experiments. ${ }^{*} p<0.05$.

amplification when performing RT-PCR with the divergent primers on genomic DNA (Figure 1(a), lower panel). LinearITCH was amplificated from both of cDNA and genomic DNA templates (Figure 1(a)). GAPDH was used as a linear control (Figure 1(a)). Thus, we confirmed that cir-ITCH is specifically amplified with divergent primers on cDNA.

\section{2. cir-ITCH Is Overexpressed and Positively Correlated with} ITCH Expression in Lung Cancer Tissues. Next, cDNA of cancer tissues and paired noncancerous tissues of 78 lung cancer patients was extracted, and then the expression level of $c i r-I T C H$ was evaluated with the divergent primer set. cirITCH was expressed at a lower level in approximately $73.08 \%$ of the lung cancer tissues compared to that of the paired noncancerous samples (Figure 1(b)). Simultaneously, we further evaluated the association between cir-ITCH expression in lung cancer tissues and clinical characteristics of lung cancer patients. As shown in Table 1, cir-ITCH expression in lung cancer tissues was significantly associated with age ( $p=0.0076)$; however, it is not correlated with other clinical characteristics including sex $(p=0.9704)$, family history ( $p=0.8437)$, smoking $(p=0.1713)$, drinking $(p=0.1568)$, tumor type $(p=0.9183)$, and TNM stage $(p=0.2531)$ in lung cancer patients. To study the correlation between cirITCH and ITCH in lung cancer, we assessed the expression of cir-ITCH in randomly selected 20 pairs of tissue samples from the 78 patients. The results showed that patients with higher cir-ITCH expression levels in lung cancer tissues had a 
TABLE 3: The sequence of the predicted miRNA binding sites on the $3^{\prime}$-UTR region of ITCH and cir-ITCH.

\begin{tabular}{lrr}
\hline MicroRNA & miRNA binding sites $3^{\prime}$-UTR & miRNA binding sites in cir-ITCH \\
\hline miRNA-7 & GUGGCCACAUGUAUAUAGUCUUCCC & UGAGGUAGUAGGUUGUAUAGUU \\
miRNA-214 & UGUAUAUGUCUUCCCUGCUGU & ACAGCAGGCACAGACAGGCAGU \\
\hline
\end{tabular}

substantial upregulation of linear ITCH $\left(R^{2}=0.33, p<0.01\right.$; Figure 1(c)).

\subsection{Characterization of cir-ITCH in Lung Cancer Cells. To} further study the role of cir-ITCH in lung cancer progression, we constructed a recombinant vector to express cir-ITCH in lung cancer cell lines according to the previous studies [15, 16]. Then, the constructed plasmid was transiently transfected into A549 and NCI-H460 cells. Next, random primers and oligo (dT) primers were used to reverse total RNA and mRNA into cDNA, respectively. In contrast to the linear products, we thought that circular products amplified with the divergent primer set would be depleted in the poly(A)-enriched RNA [28]. In our results, the expression of linear ITCH (normalized to GAPDH) showed no difference between total RNA and poly(A)-enriched RNA in both A549 cells and NCI-H460 cells (Figure 1(d)), while the expression of cirITCH was significantly decreased in poly(A)-enriched RNA compared with total RNA in these two lung cancer cell lines (Figure 1(d)).

Circular RNAs are resistant toward exonucleases for the reason of lacking free ends $[29,30]$. To further confirm the circular characteristics of cir-ITCH in A549 cells and NCI$\mathrm{H} 460$ cells, the enzyme RNase $\mathrm{R}$, a highly processive $3^{\prime}$ to $5^{\prime}$ exoribonuclease that does not react on circular RNAs, was used to digest total RNA $[31,32]$, and then we performed RTPCR to evaluate linear ITCH and cir-ITCH expression. As expected, in contrast to the linear ITCH, the predicted cirITCH was resistant to the RNase R treatment (Figure 1(e)).

3.4. Interaction between cir-ITCH and miRNA. miR-7 and miR-214 can bind to the $3^{\prime}$-untranslated region (UTR) of ITCH and cir-ITCH [16], and binding sites of these two miRNAs were presented in Table 3. The stability of cir-ITCH was firstly investigated with the presence of miRNA mimic or inhibitor via actinomycin D assay. A549 and NCI-H460 cells were cotransfected with the cir-ITCH plasmid and miRNA mimic or inhibitor, respectively. And, then, cells were treated with actinomycin D, a transcription inhibitor. Total RNA was extracted at indicated time points and the relative expression of cir-ITCH was evaluated. There was almost no change in cir-ITCH levels in both A549 cells and NCI-H460 cells with miRNA mimic or inhibitor treatment (Figures 2(a) and 2(d)), while the cir-ITCH levels in cells transfected with empty vector remained only 20-30\% (Figures 2(b) and 2(e)), which were significantly lower than that in cells transfected with the cir-ITCH plasmid $(p<0.01)$. These results suggest that miR7 and miR-214 can degrade cir-ITCH in lung cancer cells.

Next, the ITCH binding sequences of miR-7 and miR214 were inserted into psiCHECK-2 vector, respectively. The constructed luciferase reporter of miR-7 or miR-214 and cir-ITCH plasmid were transiently cotransfected into lung cancer cells, and the luciferase activity was subsequently detected. In both A549 cells (Figure 3(a)) and NCI-H460 cells (Figure 3(b)) transfected with empty vector (control of cirITCH plasmid), luciferase activity was significantly decreased in a concentration-dependent manner with the presence of miR-7/miR-214 mimic. However, there were no significant differences in luciferase activity of $\mathrm{miR}-7 / \mathrm{miR}-214 \mathrm{mimic}$ in cells with cir-ITCH hyperexpression (Figures 3(a) and 3(b)). Thus, cir-ITCH can act as sponge of ITCH to interact with miR-7 and miR-214 in lung cancer cells.

3.5. cir-ITCH Inhibits the Activation of Wnt/ $\beta$-Catenin Signaling Pathway. ITCH protein promotes the degradation of phosphorylated Dvl2, which is an important regulator for Wnt/ $\beta$-catenin signaling activation [21]. cir-ITCH, acting as a sponge of oncogenic miRNAs, can competitively inhibit these miRNAs' bind to ITCH and thus indirectly suppresses the activation of $\mathrm{Wnt} / \beta$-catenin signaling in esophageal squamous cell carcinoma [16]. To further confirm whether cir-ITCH regulates the Wnt/ $\beta$-catenin signaling pathway in lung cancer cells, we used a $\beta$-catenin/T-cell factor- (TCF-) responsive luciferase reporter assay [33]. As shown in Figure 4(a), overexpression of cir-ITCH significantly suppressed relative TCF transcriptional activity in both A549 cells and NCI-H460 cells, which suggests that cir-ITCH inhibits $\beta$ catenin expression. The expression level of $\beta$-catenin in lung cancer cells with cir-ITCH hyperexpression was further confirmed by western blotting analysis (Figure 4(b)), and it was discovered that there was an obvious decrease in $\beta$ catenin levels, while no change in Wnt3a expression was shown. Oncogene c-Myc and cell cycle regulator cyclinD1 are two important downstream targets of $\beta$-catenin [33, 34]; then, we investigated the effect of cir-ITCH on the mRNA expression of these two proteins. In lung cancer cells transfected with cir-ITCH, mRNA expression of c-Myc and cyclinD1 was significantly suppressed compared to empty vector control (Figure 4(c)).

3.6. cir-ITCH Inhibits Cellular Proliferation of Lung Cancer. cir-ITCH inhibits cell proliferation in both esophageal squamous cell carcinoma and colorectal cancer $[15,16]$. To further confirm the role of cir-ITCH in lung cancer cell proliferation, lung cancer cells were transfected with cir-ITCH with or without miR-7 and miR-214. We noticed that miR-7 and miR214 significantly promoted cell proliferation of A549 cells and NCI-H460 cells compared to simple empty vector transfection, while cell proliferation was dramatically decreased when cir-ITCH was overexpressed with the presence of miR-7 and miR-214 treatment compared to the controls. In lung cancer cells transfected with cir-ITCH with or without miR-7 and miR-214, treatment showed no significant difference (Figures 4(d) and 4(e)). 

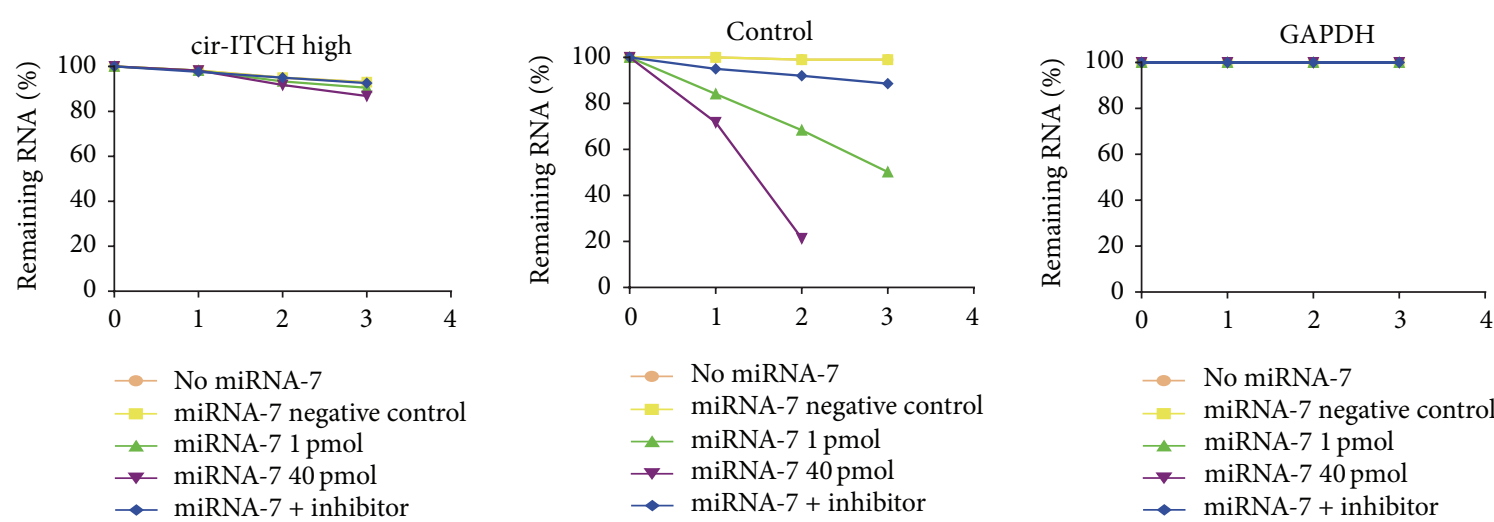

$\longrightarrow$ No miRNA-7

-1- miRNA-7 negative control

- miRNA-7 1 pmol

$\rightarrow$ miRNA-7 40 pmol

$\rightarrow$ miRNA-7 + inhibitor
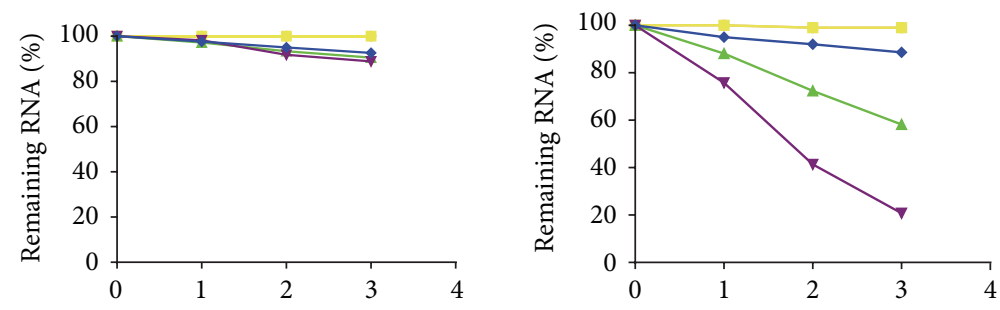

$\rightarrow$ No miRNA-214

-1- miRNA-214 negative control

— miRNA-214 1 pmol

$\rightarrow$ miRNA-214 40 pmol

$\rightarrow$ miRNA-214 + inhibitor

(a)

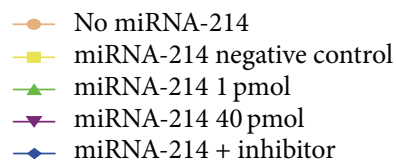

(b)

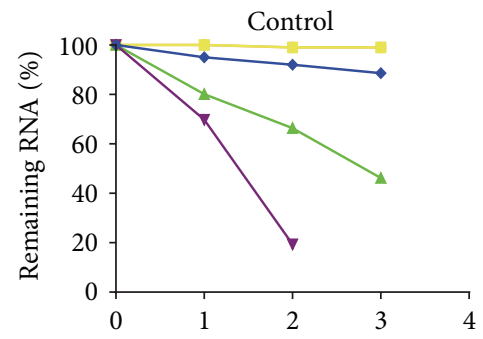

- No miRNA-7

- miRNA-7 negative control

$\simeq$ miRNA-7 1 pmol

$\rightarrow$ miRNA-7 40 pmol

$\rightarrow$ miRNA-7 + inhibitor

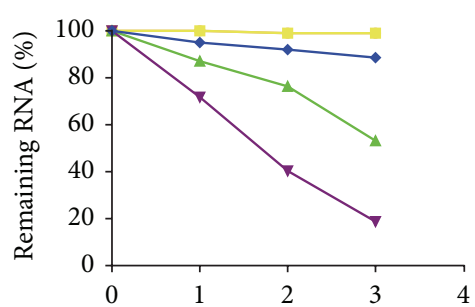

- No miRNA-214

- miRNA-214 negative control

- miRNA-214 $1 \mathrm{pmol}$

$\rightarrow$ miRNA-214 40 pmol

$\rightarrow$ miRNA-214 + inhibitor

(e)

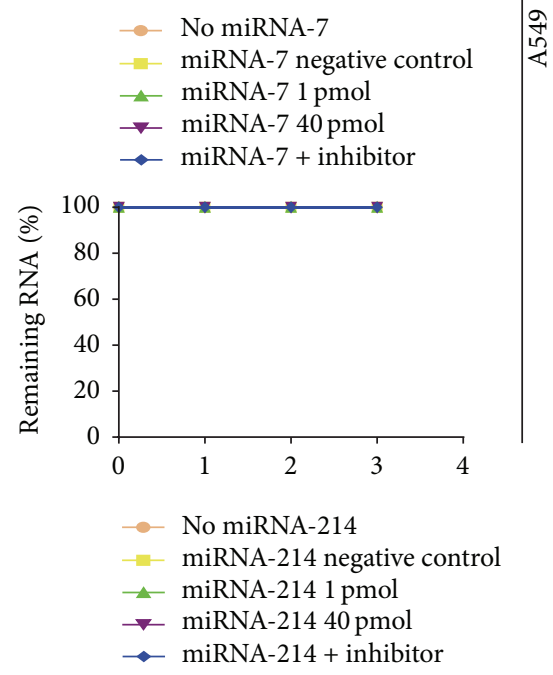

(c)

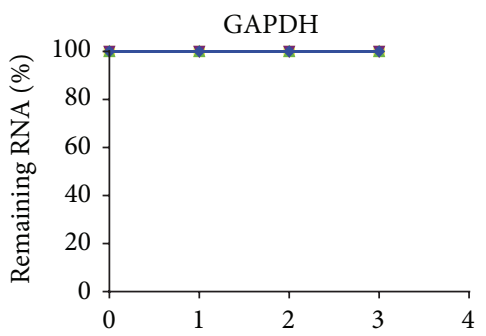

- No miRNA-7

- miRNA-7 negative control

- miRNA-7 1 pmol

$\checkmark$ miRNA-7 40 pmol

- miRNA-7 + inhibitor

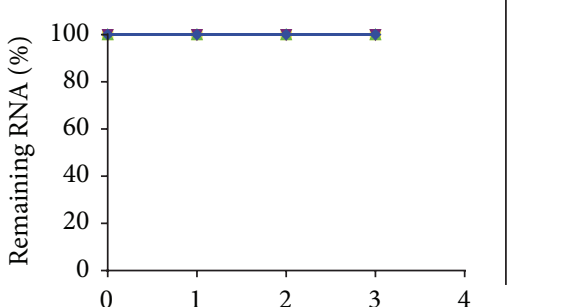

-- No miRNA-214

- miRNA-214 negative control

$\rightarrow$ miRNA-214 1 pmol

$\rightarrow$ miRNA-214 40 pmol

$\rightarrow$ miRNA-214 + inhibitor

(f)

FIGURE 2: $c$ ir-ITCH inhibits the expression of miR-7 and miR-214. A549 cells were, respectively, transfected with cir-ITCH (a) and empty control vector (b) and simultaneously treated with miR-7 (upper) and miR-214 (lower) for $24 \mathrm{~h}$. Cells were then further exposed to actinomycin $\mathrm{D}$ for 1,2 , and $3 \mathrm{~h}$. The stability of cir-ITCH mRNA was analyzed by qRT-PCR relative to $0 \mathrm{~h}$ after actinomycin D treatment. (c) GAPDH as endogenous control. ((d)-(f)) Similar to A549 cells, the stability of cir-ITCH mRNA in NCI-H460 cells was evaluated by qRT-PCR. Data are presented as mean \pm SEM, normalized to GAPDH. 

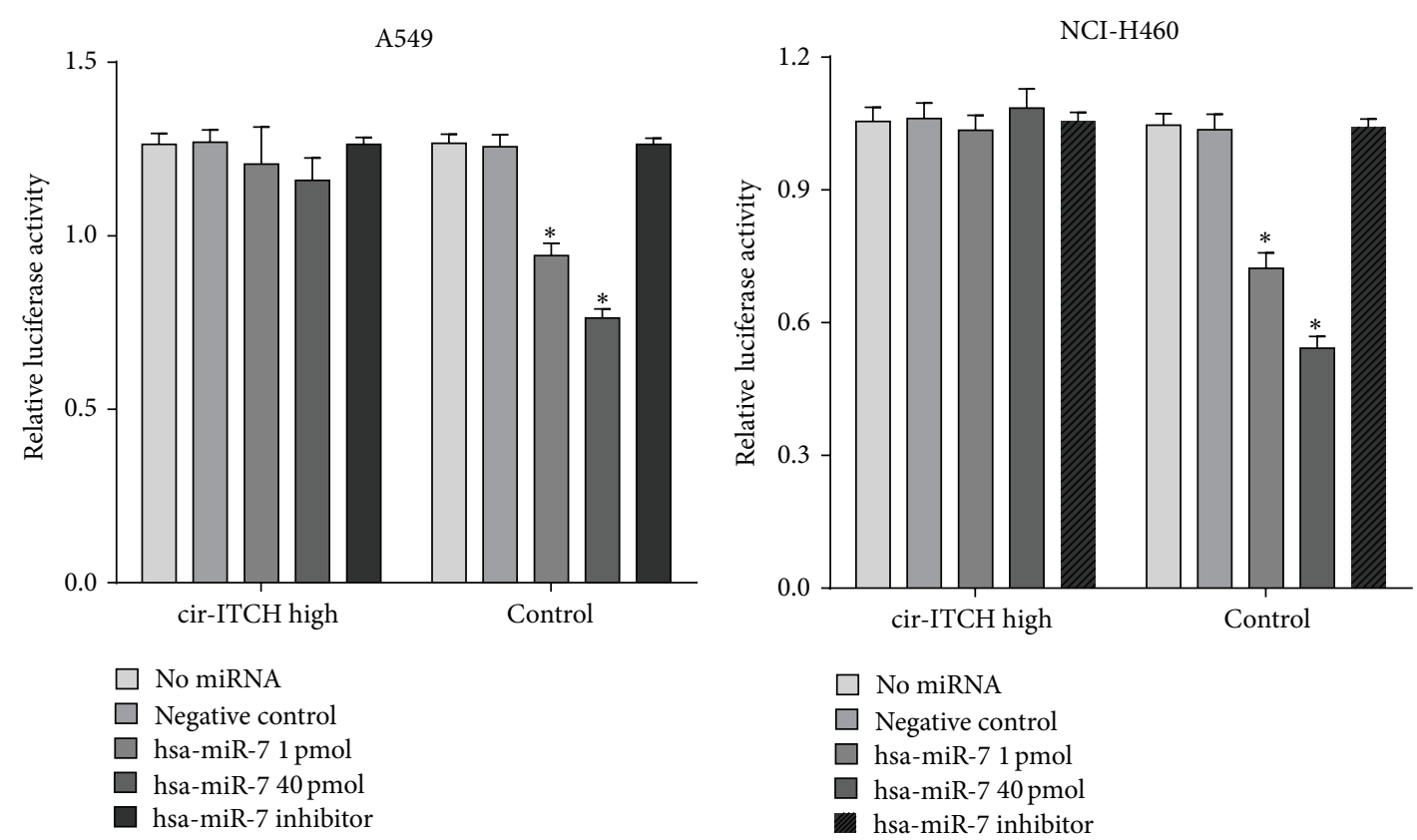

(a)
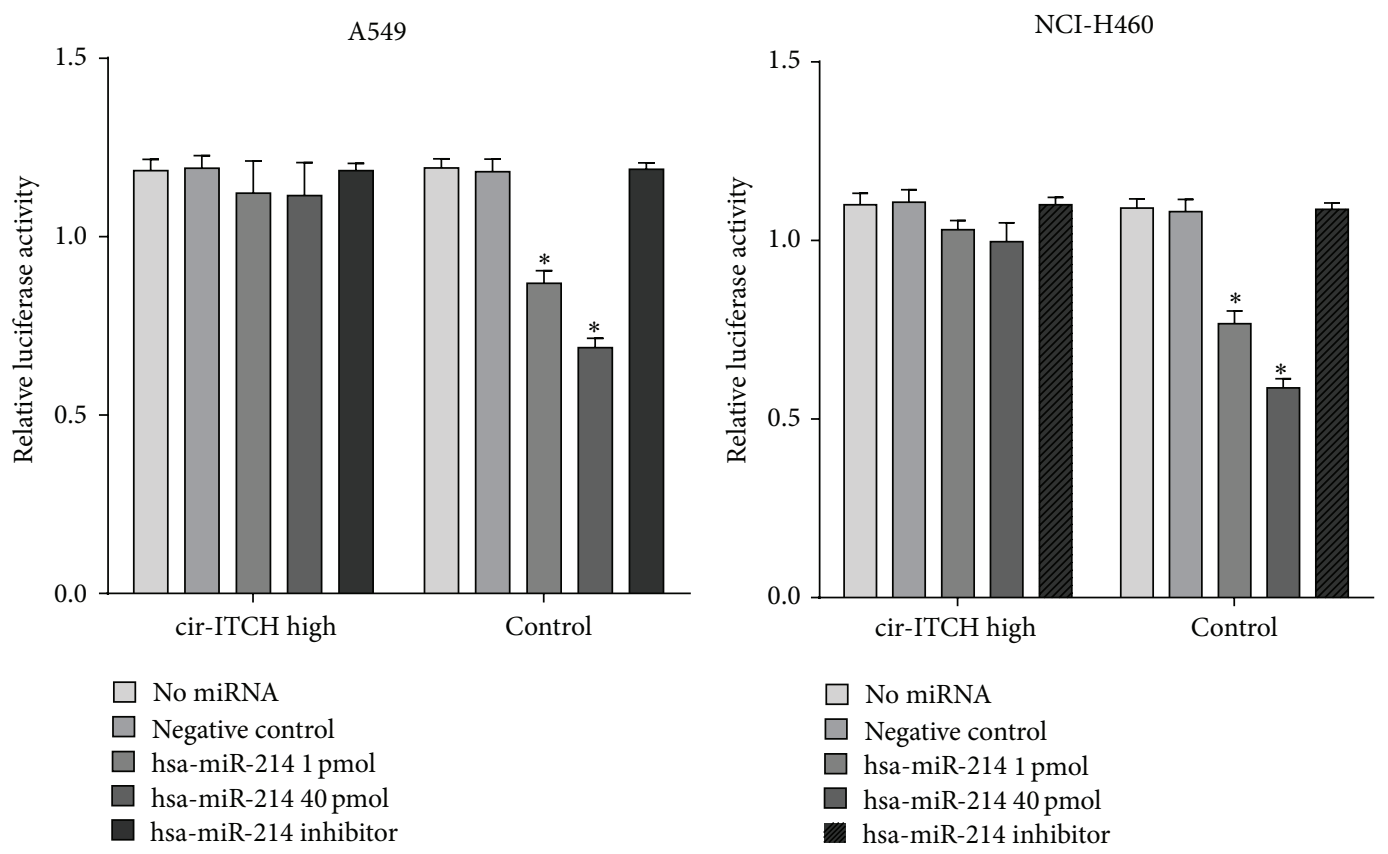

(b)

FIGURE 3: cir-ITCH acts as microRNA sponges of miR-7 and miR-214. (a) A549 (left) and NCI-H460 (right) cells were cotransfected with psiCHECK-2-ITCH constructs and cir-ITCH or empty control vector, respectively. Cells were further simultaneously treated with miR-7 mimic or inhibitor for $24 \mathrm{~h}$. Then, relative luciferase activity of psiCHECK-2-ITCH constructs was evaluated. (b) Similar to miR-7, relative luciferase activity of the psiCHECK-2-ITCH constructs in A549 and NCI-H460 cells with the presence of miR-214 was evaluated. Data are presented as mean \pm SEM from six replicates for each group. ${ }^{*} p<0.05$.

\section{Discussion}

Recently, many studies have confirmed the widespread and abundant presence of circular RNA in eukaryotic cells [3538]. Specifically, cir-ITCH has been reported to inhibit the progression of esophageal squamous cell carcinoma and colorectal cancer mainly by regulating the $\mathrm{Wnt} / \beta$-catenin pathway $[15,16]$. However, the function of cir-ITCH in lung cancer is still unclear. In this study, we compared the expression level of cir-ITCH in lung cancer issues by using a TaqMan-based RT-PCR and found that cir-ITCH was dramatically decreased in lung cancer tissues, indicating cirITCH may play a role in regulating lung cancer progression. 


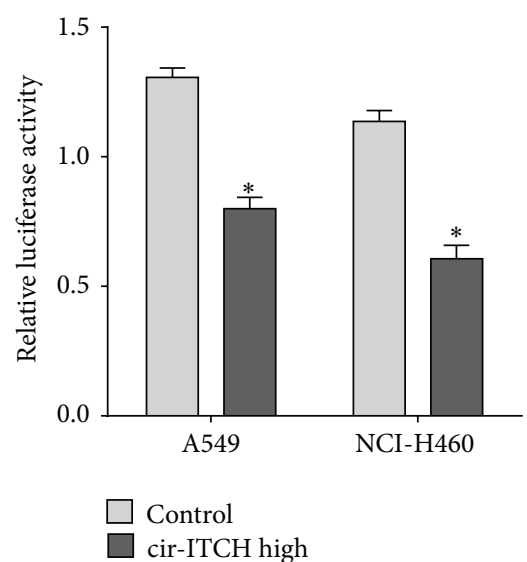

(a)

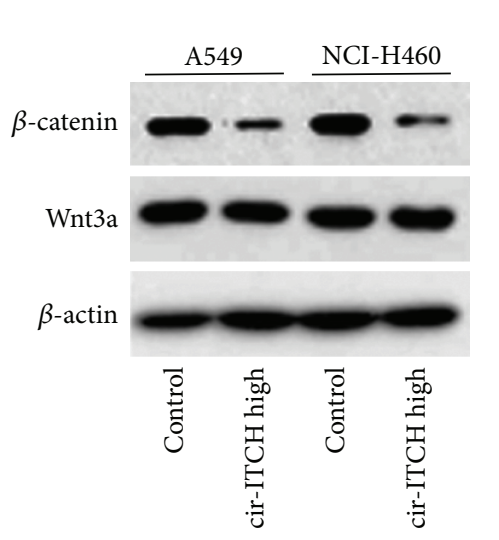

(b)

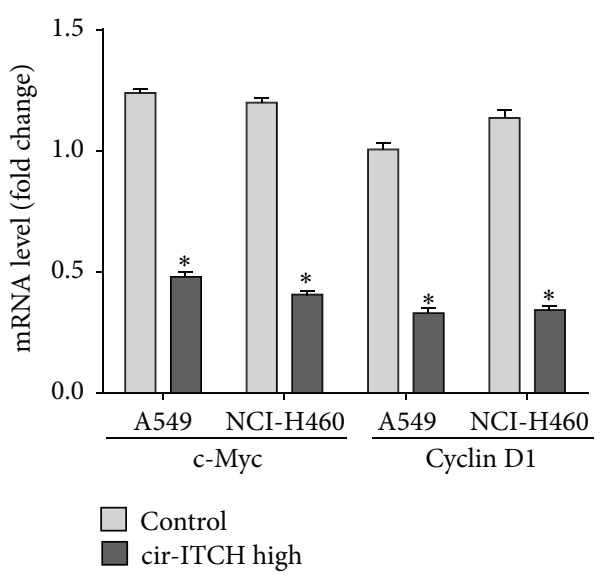

(c)

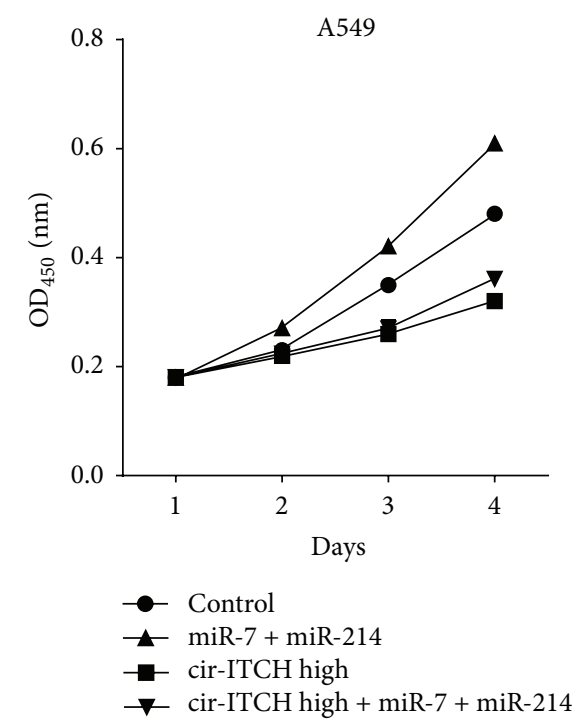

(d)

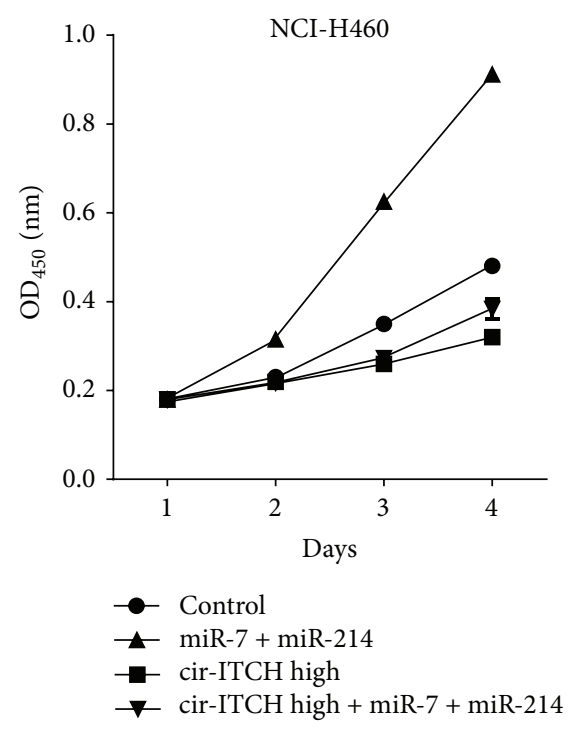

(e)

FIGURE 4: cir-ITCH can regulate the Wnt/ $\beta$-catenin signaling pathway and cellular proliferation of lung cancer cells. (a) A549 and NCI-H460 cells were transfected with cir-ITCH or empty control vector; then, a $\beta$-catenin/T-cell factor- (TCF-) responsive luciferase reporter assay was performed. The luciferase activity was normalized to the Renilla luciferase activity. (b) The protein levels of Wnt3a and $\beta$-catenin were assessed in A549 cells and NCI-H460 cells by western blotting. $\beta$-actin was used as endogenous control. (c) The mRNA level of c-Myc and cyclinD1 in A549 cells and NCI-H460 cells was detected by qRT-PCR after being transfected with cir-ITCH or empty control vector. (d) A549 and (e) NCI-H460 cells were seeded in 96-well plates after being transfected with cir-ITCH and empty control vector, and cell proliferation was detected daily for 3 days by using the CCK- 8 assay. Data are presented as mean \pm SEM from six replicates for each group. ${ }^{*} p<0.05$.

cir-ITCH, located on chromosome 20q11.22, is aligned in a sense orientation to the known protein-coding gene, ITCH, a member of the E3 ubiquitin ligases [16]. Correlation analysis showed that cir-ITCH expression in lung cancer tissues was not correlated with clinicopathological characteristics except age. This is likely to be because the incidence of lung cancer increases with age, particularly after the age of 60 [39], and we still need to confirm this result in a larger sample population. As parental gene of $c i r-I T C H$, ITCH also decreased in lung cancer patients and positively correlated with $\operatorname{cir}$-ITCH. Hence, we speculated that ITCH may play a tumor suppressive role in lung cancer, and there is a possible connection between ITCH and cir-ITCH. ITCH involved in cancer progression mainly depends on its ability to regulate protein stability [40-42] and the target proteins including p63 [43], p73 [44], Notch1 [45], Dvl2 [21], RASSF5 [42], and LATS1 [41]. These proteins usually associated with tumor formation and chemosensitivity serve as either tumor suppressor or enhancer; thus, the role of ITCH in tumor progression is complicated.

Concerning the function of $\operatorname{cir}-I T C H$, it is speculated that cir-ITCH serves as epigenetic miRNA sponges to competitively block the bind between miRNA and ITCH. Previous research has shown that miR-216b, miR-17, miR-214, miR-7, miR-20a, and miR-128 could bind to the $3^{\prime}$-UTR of ITCH and cir-ITCH $[15,16]$. In our study, we found that miR7, miR-214, and miR-128 (data not shown) decreased ITCH expression by binding to its $3^{\prime}$-UTR in lung cancer cell lines, 
and cir-ITCH acts as a sponge for miR-7 and miR-214, except miR-128. These results were not fully consistent with study in cell lines of esophageal squamous cell carcinoma [16], in which $c i r-I T C H$ acts as a sponge for five miRNAs: miR-216b, miR-17, miR-214, miR-7, and miR-128. The reason may be owing to the difference of cancer origin, as the expression and function of circRNAs occupy tissue- and developmentspecific properties [11]. cir-ITCH also did not act as a sponge for miR-214 but for miR-20a in colorectal cancer [15]. As two oncogenic miRNAs, miR-7 and miR214 are involved in the progression of many cancers including lung cancer $[22,23$, 46-49]. In lung cancer cells, we found that miR-7 and miR214 promoted cell proliferation; this activity was totally abrogated with ectopic cir-ITCH hyperexpression. Thus, cir-ITCH is involved in lung cancer progression by interacting miRNAs. However, with regard to other tumor-related activities, like migration, invasion, and colony formation, the antitumor effects of cir-ITCH in lung cancer still need to be further investigated.

ITCH is crucial in the control of proteasome degradation of Dvl2, which inhibits Wnt/ $\beta$-catenin signaling [21, 50]. Deregulated Wnt $/ \beta$-catenin signaling with cancers has been well documented in tumor initiation, progression, and metastasis, including lung cancer [18-20, 51, 52]. Blocking $\beta$-catenin signaling for cancer treatment has thus generated significant interests [53]. The beneficial effect of nonsteroidal anti-inflammatory drugs (NSAIDs) in cancer prevention and therapy has been attributed partially to the perturbation of $\beta$ catenin signaling [54]. In our study, cir-ITCH inhibits Wnt/ $\beta$ catenin signaling in lung cancer cells with the evidence that hyperexpression of $c i r-I T C H$ significantly suppressed relative TCF transcriptional activity in $\beta$-catenin/TCF-responsive luciferase reporter assay. This result was further confirmed by western blotting analysis.

At last, we examined the impacts of cir-ITCH on two important downstream targets of $\mathrm{Wnt} / \beta$-catenin pathway, c-Myc and cyclinD1, which are continually overexpressed in many cancers and have crucial roles in regulating cell growth, apoptosis, and differentiation [55]. Hyperexpression of cir-ITCH significantly suppressed the mRNA expression of c-Myc and cyclinD1 in lung cancer cells. Combined with previous studies $[15,16]$, we are able to conclude that cirITCH has an antitumor role in lung cancer by controlling miRNA activity, which increases the concentration of ITCH and results in suppression of the canonical $\mathrm{Wnt} / \beta$-catenin pathway.

In conclusion, our study demonstrates that the cir-ITCH acts as a sponge for miR-7 and miR-214, promotes the expression of their target gene ITCH, and thus regulates lung cancer cell proliferation by indirectly inhibiting the activation of Wnt/ $\beta$-catenin pathway. Further characterization of the function of circular RNAs in cancer progression will have great implication for the development of new RNA-based cancer diagnosis and therapy.

\section{Competing Interests}

The authors declare no conflict of interests.

\section{Acknowledgments}

This study was supported by the National Natural Science Foundation of China (Grant no. 81000032).

\section{References}

[1] W. Chen, R. Zheng, P. D. Baade et al., "Cancer statistics in China, 2015," CA: A Cancer Journal for Clinicians, vol. 66, no. 2, pp. 115132, 2016.

[2] T. Kosaka, Y. Yatabe, H. Endoh, H. Kuwano, T. Takahashi, and T. Mitsudomi, "Mutations of the epidermal growth factor receptor gene in lung cancer: biological and clinical implications," Cancer Research, vol. 64, no. 24, pp. 8919-8923, 2004.

[3] Q.-Y. Hong, G.-M. Wu, G.-S. Qian et al., "Prevention and management of lung cancer in China," Cancer, vol. 121, no. 17, pp. 3080-3088, 2015.

[4] I. Petersen and S. Petersen, "Towards a genetic-based classification of human lung cancer," Analytical Cellular Pathology, vol. 22, no. 3, pp. 111-121, 2001.

[5] L. G. Collins, C. Haines, R. Perkel, and R. E. Enck, "Lung cancer: diagnosis and management," American Family Physician, vol. 75, no. 1, pp. 56-63, 2007.

[6] R. L. Siegel, K. D. Miller, and A. Jemal, “Cancer statistics, 2015," CA-A Cancer Journal for Clinicians, vol. 65, no. 1, pp. 5-29, 2015.

[7] S. Memczak, M. Jens, A. Elefsinioti et al., "Circular RNAs are a large class of animal RNAs with regulatory potency," Nature, vol. 495, no. 7441, pp. 333-338, 2013.

[8] J. M. Nigro, K. R. Cho, E. R. Fearon et al., "Scrambled exons," Cell, vol. 64, no. 3, pp. 607-613, 1991.

[9] W. R. Jeck, J. A. Sorrentino, K. Wang et al., "Circular RNAs are abundant, conserved, and associated with ALU repeats," RNA, vol. 19, no. 2, pp. 141-157, 2013.

[10] J. Salzman, C. Gawad, P. L. Wang, N. Lacayo, and P. O. Brown, "Circular RNAs are the predominant transcript isoform from hundreds of human genes in diverse cell types," PLOS ONE, vol. 7, no. 2, Article ID e30733, 2012.

[11] J. Salzman, R. E. Chen, M. N. Olsen, P. L. Wang, and P. O. Brown, "Cell-type specific features of circular RNA expression," PLoS Genetics, vol. 9, no. 9, Article ID e1003777, 2013.

[12] T. B. Hansen, T. I. Jensen, B. H. Clausen et al., "Natural RNA circles function as efficient microRNA sponges," Nature, vol. 495, no. 7441, pp. 384-388, 2013.

[13] M. S. Ebert, J. R. Neilson, and P. A. Sharp, "MicroRNA sponges: competitive inhibitors of small RNAs in mammalian cells," Nature Methods, vol. 4, no. 9, pp. 721-726, 2007.

[14] A. Bachmayr-Heyda, A. T. Reiner, K. Auer et al., "Correlation of circular RNA abundance with proliferation-exemplified with colorectal and ovarian cancer, idiopathic lung fibrosis, and normal human tissues," Scientific Reports, vol. 5, article 8057, 2015.

[15] G. Huang, H. Zhu, Y. Shi, W. Wu, H. Cai, and X. Chen, "CirITCH plays an inhibitory role in colorectal cancer by regulating the Wnt/ $\beta$-Catenin Pathway," PLoS ONE, vol. 10, no. 6, article e0131225, 2015.

[16] H. Liu, J. Yin, H. Wang et al., "FOXO3a modulates WNT/ $\beta$ catenin signaling and suppresses epithelial-to-mesenchymal transition in prostate cancer cells," Cellular Signalling, vol. 27, no. 3, pp. 510-518, 2015. 
[17] P. Li, S. Chen, H. Chen et al., "Using circular RNA as a novel type of biomarker in the screening of gastric cancer," Clinica Chimica Acta, vol. 444, pp. 132-136, 2015.

[18] X. Chen, X. Song, W. Yue et al., "Fibulin-5 inhibits Wnt $/ \beta$ catenin signaling in lung cancer," Oncotarget, vol. 6, no. 17, pp. 15022-15034, 2015.

[19] O. Aguilera, A. Muñoz, M. Esteller, and M. F. Fraga, "Epigenetic alterations of the Wnt/ $\beta$-catenin pathway in human disease," Endocrine, Metabolic and Immune Disorders-Drug Targets, vol. 7, no. 1, pp. 13-21, 2007.

[20] L. Serman, T. N. Martic, A. Serman, and S. Vranic, "Epigenetic alterations of the Wnt signaling pathway in cancer: a mini review," Bosnian Journal of Basic Medical Sciences, vol. 14, no. 4, pp. 191-194, 2014.

[21] W. Wei, M. Li, J. Wang, F. Nie, and L. Li, “The E3 ubiquitin ligase ITCH negatively regulates canonical Wnt signaling by targeting dishevelled protein," Molecular and Cellular Biology, vol. 32, no. 19, pp. 3903-3912, 2012.

[22] Y.-T. Chou, H.-H. Lin, Y.-C. Lien et al., "EGFR promotes lung tumorigenesis by activating miR-7 through a Ras/ERK/Myc pathway that targets the Ets2 transcriptional repressor ERF," Cancer Research, vol. 70, no. 21, pp. 8822-8831, 2010.

[23] H. Salim, N. S. Akbar, D. Zong et al., "MiRNA-214 modulates radiotherapy response of non-small cell lung cancer cells through regulation of p38MAPK, apoptosis and senescence," British Journal of Cancer, vol. 107, no. 8, pp. 1361-1373, 2012.

[24] U. Lehmann and H. Kreipe, "Real-time PCR analysis of DNA and RNA extracted from formalin-fixed and paraffinembedded biopsies," Methods, vol. 25, no. 4, pp. 409-418, 2001.

[25] M. Danan, S. Schwartz, S. Edelheit, and R. Sorek, "Transcriptomewide discovery of circular RNAs in Archaea," Nucleic Acids Research, vol. 40, no. 7, pp. 3131-3142, 2012.

[26] H. Wu, J. Zheng, J. Deng et al., "A genetic polymorphism in lincRNA-uc003opf.1 is associated with susceptibility to esophageal squamous cell carcinoma in Chinese populations," Carcinogenesis, vol. 34, no. 12, pp. 2908-2917, 2013.

[27] B. Xie, J. Zhou, G. Shu et al., "Restoration of klotho gene expression induces apoptosis and autophagy in gastric cancer cells: Tumor suppressive role of klotho in gastric cancer," Cancer Cell International, vol. 13, article 18, 2013.

[28] Y. Liu, H. Cui, W. Wang et al., "Construction of circular miRNA sponges targeting miR-21 or miR-221 and demonstration of their excellent anticancer effects on malignant melanoma cells," International Journal of Biochemistry and Cell Biology, vol. 45, no. 11, pp. 2643-2650, 2013.

[29] H. Suzuki and T. Tsukahara, "A view of pre-mRNA splicing from RNase R resistant RNAs," International Journal of Molecular Sciences, vol. 15, no. 6, pp. 9331-9342, 2014.

[30] Q. Vicens and E. Westhof, "Biogenesis of circular RNAs," Cell, vol. 159, no. 1, pp. 13-14, 2014.

[31] H. A. Vincent and M. P. Deutscher, "Substrate recognition and catalysis by the exoribonuclease RNase R," The Journal of Biological Chemistry, vol. 281, no. 40, pp. 29769-29775, 2006.

[32] H. A. Vincent and M. P. Deutscher, "Insights into how RNase R degrades structured RNA: analysis of the nuclease domain," Journal of Molecular Biology, vol. 387, no. 3, pp. 570-583, 2009.

[33] M. J. Ripple, A. P. Struckhoff, J. Trillo-Tinoco et al., "Activation of c-Myc and cyclin D1 by JCV T-Antigen and $\beta$-Catenin in colon cancer," PLoS ONE, vol. 9, no. 9, Article ID e106257, 2014.
[34] S. Zhang, Y. Li, Y. Wu, K. Shi, L. Bing, and J. Hao, "Wnt/ $\beta$ catenin signaling pathway upregulates $c$-Myc expression to promote cell proliferation of P19 teratocarcinoma cells," Anatomical Record, vol. 295, no. 12, pp. 2104-2113, 2012.

[35] P. L. Wang, "Circular RNA is expressed across the eukaryotic tree of life," PLoS ONE, vol. 9, no. 6, Article ID e90859, 2014.

[36] E. Zlotorynski, "Non-coding RNA: circular RNAs promote transcription," Nature Reviews Molecular Cell Biology, vol. 16, no. 4, p. 206, 2015.

[37] Z. J. Zhao and J. Shen, "Circular RNA participates in the carcinogenesis and the malignant behavior of cancer," RNA Biology, 2015.

[38] S. Qu, X. Yang, X. Li et al., "Circular RNA: a new star of noncoding RNAs," Cancer Letters, vol. 365, no. 2, pp. 141-148, 2015.

[39] A. Zagryazhskaya and B. Zhivotovsky, "MiRNAs in lung cancer: a link to aging," Ageing Research Reviews, vol. 17, pp. 54-67, 2014.

[40] M. Rossi, S. Inoue, R. Walewska et al., "Caspase cleavage of Itch in chronic lymphocytic leukemia cells," Biochemical and Biophysical Research Communications, vol. 379, no. 3, pp. 659664, 2009.

[41] K. C. Ho, Z. Zhou, Y. M. She et al., "Itch E3 ubiquitin ligase regulates large tumor suppressor 1 stability [corrected]," Proceedings of the National Academy of Sciences of the United States of America, vol. 108, no. 12, pp. 4870-4875, 2011.

[42] R. Suryaraja, M. Anitha, K. Anbarasu, G. Kumari, and S. Mahalingam, "The E3 ubiquitin ligase itch regulates tumor suppressor protein RASSF5/NORE1 stability in an acetylationdependent manner," Cell Death and Disease, vol. 4, no. 3, article e565, 2013.

[43] M. Rossi, R. I. Aqeilan, M. Neale et al., "The E3 ubiquitin ligase Itch controls the protein stability of p63," Proceedings of the National Academy of Sciences of the United States of America, vol. 103, no. 34, pp. 12753-12758, 2006.

[44] M. Rossi, V. De Laurenzi, E. Munarriz et al., "The ubiquitinprotein ligase Itch regulates p73 stability," The EMBO Journal, vol. 24, no. 4, pp. 836-848, 2005.

[45] L. E. Matesic, D. C. Haines, N. G. Copeland, and N. A. Jenkins, "Itch genetically interacts with Notch1 in a mouse autoimmune disease model," Human Molecular Genetics, vol. 15, no. 24, pp. 3485-3497, 2006.

[46] H. Xu, S. Guo, W. Li, and P. Yu, "The circular RNA Cdrlas, via miR-7 and its targets, regulates insulin transcription and secretion in islet cells," Scientific Reports, vol. 5, Article ID 12453, 2015.

[47] K. Xu, Z. Chen, C. Qin, and X. Song, "miR-7 inhibits colorectal cancer cell proliferation and induces apoptosis by targeting XRCC2," OncoTargets and Therapy, vol. 7, pp. 325-332, 2014.

[48] Z.-J. Zou, L. Fan, L. Wang et al., "miR-26a and miR-214 downregulate expression of the PTEN gene in chronic lymphocytic leukemia, but not PTEN mutation or promoter methylation," Oncotarget, vol. 6, no. 2, pp. 1276-1285, 2015.

[49] X. Yu, A. Luo, Y. Liu et al., "MiR-214 increases the sensitivity of breast cancer cells to tamoxifen and fulvestrant through inhibition of autophagy," Molecular Cancer, vol. 14, article 208, 2015.

[50] Y. Zhang, F. Wang, L. Han et al., "GABARAPL1 negatively regulates wnt $/ \beta$-catenin signaling by mediating Dvl 2 degradation through the autophagy pathway," Cellular Physiology and Biochemistry, vol. 27, no. 5, pp. 503-512, 2011. 
[51] M. Ono, P. Yin, A. Navarro et al., "Paracrine activation of $\mathrm{WNT} / \beta$-catenin pathway in uterine leiomyoma stem cells promotes tumor growth," Proceedings of the National Academy of Sciences of the United States of America, vol. 110, no. 42, pp. 17053-17058, 2013.

[52] N. Bouteille, K. Driouch, P. E. Hage et al., "Inhibition of the Wnt/B-catenin pathway by the WWOX tumor suppressor protein," Oncogene, vol. 28, no. 28, pp. 2569-2580, 2009.

[53] B. T. MacDonald, K. Tamai, and X. He, "Wnt/ $\beta$-catenin signaling: components, mechanisms, and diseases," Developmental Cell, vol. 17, no. 1, pp. 9-26, 2009.

[54] J. Shao, C. Jung, C. Liu, and H. Sheng, "Prostaglandin E2 stimulates the $\beta$-catenin/T cell factor-dependent transcription in colon cancer," The Journal of Biological Chemistry, vol. 280, no. 28, pp. 26565-26572, 2005.

[55] F. Wang, J. Xia, N. Wang, and H. Zong, "miR-145 inhibits proliferation and invasion of esophageal squamous cell carcinoma in part by targeting c-Myc," Onkologie, vol. 36, no. 12, pp. 754-758, 2013. 


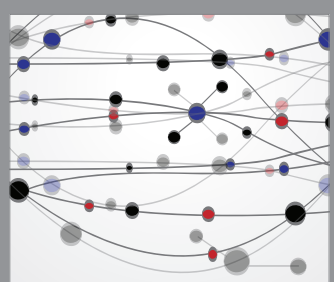

The Scientific World Journal
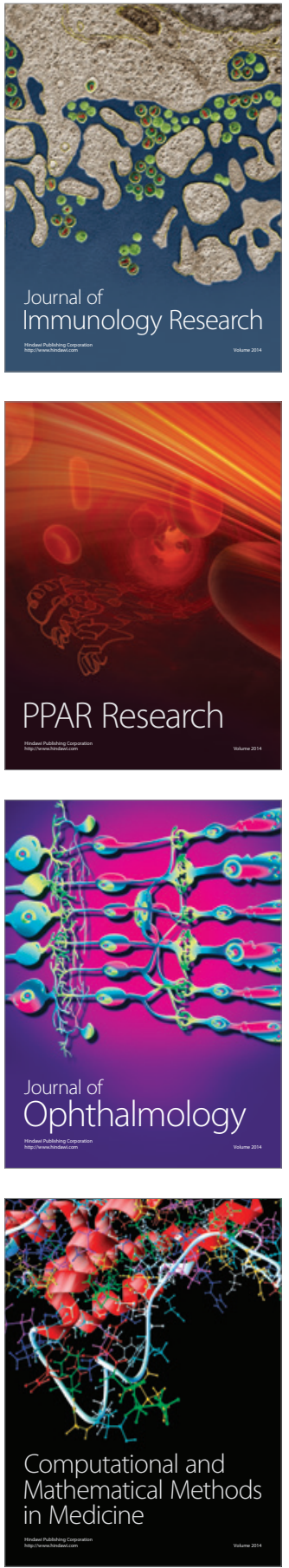

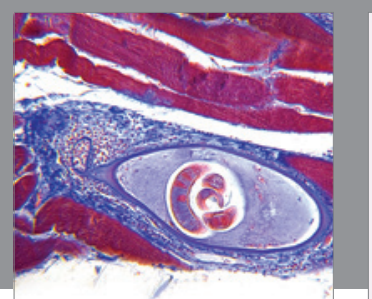

Gastroenterology Research and Practice

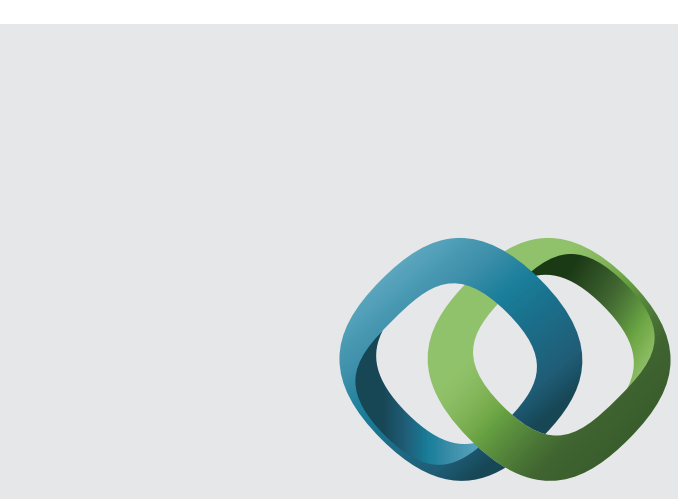

\section{Hindawi}

Submit your manuscripts at

http://www.hindawi.com
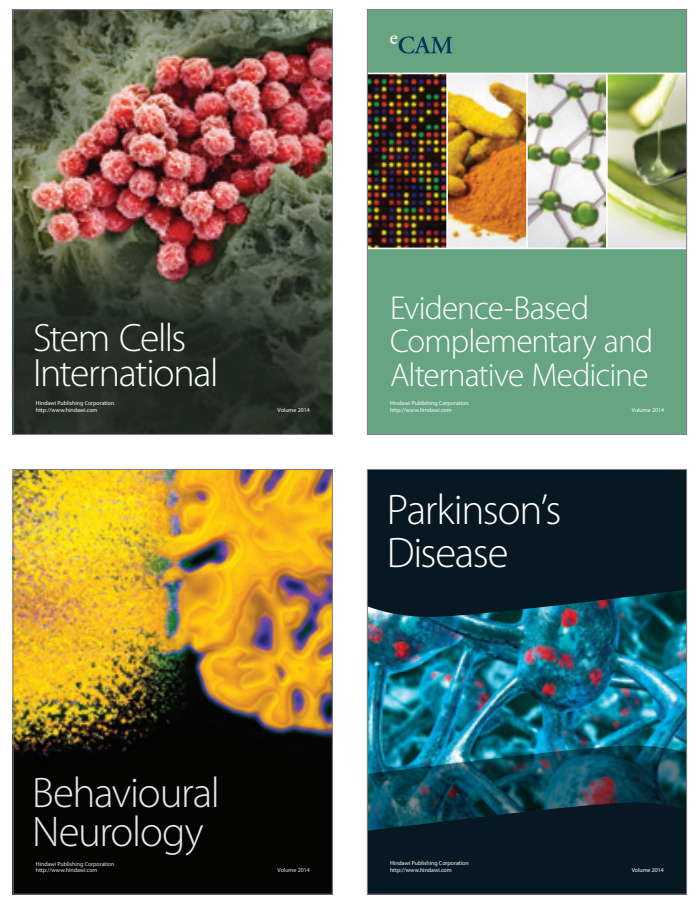
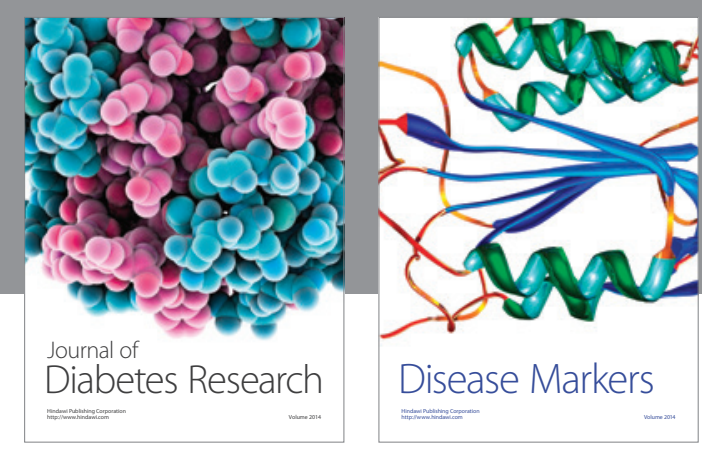

Disease Markers
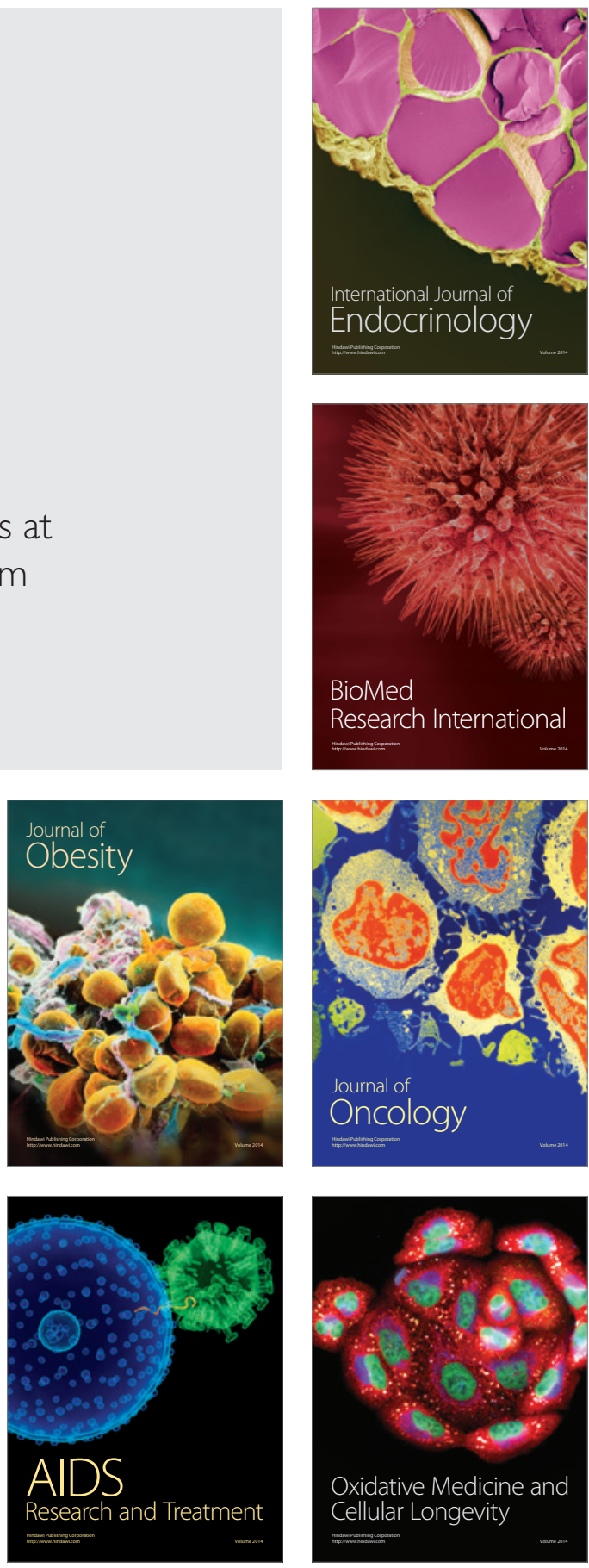